\title{
Wave localization in binary isotopically disordered one-dimensional harmonic chains with impurities having arbitrary cross section and concentration
}

\author{
K.A. Snyder \\ Materials and Construction Division, National Institute of Standards and Technology \\ T.R. Kirkpatrick \\ Institute for Physical Science and Technology and Department of Physics, University of Maryland
}

(Dated: November 8, 2018)

\begin{abstract}
The localization length for isotopically disordered harmonic one-dimensional chains is calculated for arbitrary impurity concentration and scattering cross section. The localization length depends on the scattering cross section of a single scatterer, which is calculated for a discrete chain having a wavelength dependent pulse propagation speed. For binary isotopically disordered systems composed of many scatterers, the localization length decreases with increasing impurity concentration, reaching a mimimum before diverging toward infinity as the impurity concentration approaches a value of one. The concentration dependence of the localization length over the entire impurity concentration range is approximated accurately by the sum of the behavior at each limiting concentration. Simultaneous measurements of Lyapunov exponent statistics indicate practical limits for the minimum system length and the number of scatterers to achieve representative ensemble averages. Results are discussed in the context of future investigations of the time-dependent behavior of disordered anharmonic chains.

PACS numbers: 45.05.+x, 45.30.+s, 46.40.Cd, 62.40.+d
\end{abstract}

\section{INTRODUCTION}

The length scale over which Anderson localization ${ }^{1}$ occurs in harmonic disordered chains can be expressed as a function of the ensemble-averaged system resistivity ${ }^{2.3}$. Using scaling arguments, ${ }^{3}$ the ensemble-averaged resistivity of harmonic systems having binary isotopic disorder (single-valued impurities) can be expressed as a function of the scattering cross section of a single impurity and the impurity concentration. Binary isotopically disodered harmonic chains also have the property that in the limit that the impurity concentration approaches unity, the system is once again "pure," and the Anderson localization length diverges toward infinity. As a result, the Anderson localization length passes through a minimum at intermediate concentrations ${ }^{4}$. These properties of a binary isotopically disordered harmonic chain (BIDHC) make these systems both tractable and interesting.

In this work, an approximation is developed for the localization length of a BIDHC with arbitrary impurity cross section and concentration. In addition, the result incorporates the discrete nature of a classical chain. Similar studies have been performed previously, but with important differences. Bourbonnais and Maynard $\frac{5}{5}$ studied energy transport in one-dimensional systems having isotopic disorder, but the impurity masses were not singlevalued. The results of Azbel and Soven ${ }^{6}$ on quantum systems having binary isotopic disorder were based on a continuum solution for impurity locations constrained to exist on lattice sites. Although the Azbel and Soven result applied to short wavelengths that may exist between the impurities, the results do not incorporate the additional features of a discrete mechanical system.

This study serves as an introduction to future work on energy transport in binary isotopically disordered anharmonic chains. These anharmonic chains serve as a useful model for, among other things, line width broadening ${ }^{7.8 .9}$. To perform that work, numerical integration will used to study the time-dependent nature of these systems. To be practical, the initial conditions will require sufficiently short wavelengths and high impurity concentrations to keep integration times manageable. In addition, the results of the study will incorporate the discrete nature of the mechanic chains so that these effects can accounted for in the results.

In this study, the energy localization in a BIDHC is studied for arbitrary displacement wavelength, impurity concentration and scattering cross section. Disorder is effected by changing randomly selected masses by a fixed amount. A continuum Kronig-Penney (KP) mode 10 is used to develop a general expression for scattering cross section, and the continuum impurity impedance is corrected for wavelength dependent pulse propagation speed in discrete systems. The resulting expression is verified by direct numerical integration. The localization length of systems with strong scatterers is calculated using both the continuum KP model and the MacKinnon and Kramer 11 (MK) method. The distribution of Lyapunov exponents is studied using the continuum KP model, and the minimum requirements are found for system length and number of scatterers to achieve proper scaling statistics. The localization length concentration dependence is studied using the MK method, and an analytical expression is found for its behavior. For arbitrary impurity concentration and impurity cross section, the localization length in a BIDHC can be approximated by invoking a simple ansatz based on an analogy to electrical systems. The result is accurate for systems having 
displacement wavelengths at least four lattice spacings long.

\section{MODEL SYSTEM}

The physical model used here is the harmonic version of the Fermi-Pasta-Ulam (FPU) 12 chain that is composed of discrete springs and masses. The masses $m_{i}$ are on a lattice with spacing $a$ and interact via nearest neighbor springs with force constant $K$. Disorder is effected by changing the background mass $m_{\circ}$ by a fixed amount $m_{+}$ with probability $c$. To simplify the results, all lengths are scaled by the lattice spacing $a$.

For a system composed of $N$ masses, each characterized by a displacement $x_{i}$ about the equilibrium location and a momentum $p_{i}$, the Hamiltonian is separable:

$$
H=\frac{1}{2} \sum_{i}^{N} \frac{p_{i}^{2}}{m_{i}}+K\left(x_{i+1}-x_{i}\right)^{2}
$$

The real space equation of motion is

$$
\frac{m_{i}}{K} \ddot{x}_{i}=x_{i+1}-2 x_{i}+x_{i-1}
$$

The Fourier transform leads to the corresponding equation of motion for the energy eigenstate amplitudes $u_{i}(\omega)$ :

$$
u_{i+1}=\left[2-\frac{\omega^{2} m_{i}}{K}\right] u_{i}-u_{i-1}
$$

This is the corresponding Anderson tight-binding model for the chain.

\section{A. Discrete Analysis}

The time-dependent properties of the system were determined by numerical time integration of Eqn. 2 using a fourth-order symplectic integrator algorithm (SIA4) for separable Hamiltonians. The coefficients were taken from Candy and Rozmus ${ }^{13}$, and the time step was $1 / 200$ of the natural period.

The localization length was calculated from Eqn. 3 using the method of MacKinnon and Kramer $(\mathrm{MK})^{11}$. This method exploits the statistical properties of the $u_{i}$ so that periodic rescaling can be used to improve overall statistics.

\section{B. Continuum Analysis}

A Kronig-Penney mode ${ }^{10}$ is used to develop an expression for the scattering cross section of an impurity and to study the statistics of the scaling parameter ${ }^{3}$. The continuum system analogous to the discrete chain is a homogeneous elastic rod having mass density $\mu$ and Youngs modulus $E$. In the absence of impurities, a longitudinal displacement amplitude $\psi(x, t \mid \omega)$ with angular velocity $\omega$ will propagate down the rod with longitudinal velocity $c_{l}=\sqrt{E / \mu}$.

A harmonic oscillator impurity located at $x^{\prime}$ will give rise to a reactive force due to the impurity impedance $Z$ for a wave with angular velocity $\omega$ :

$$
\left[\mu \frac{\partial^{2}}{\partial t^{2}}-E \frac{\partial^{2}}{\partial x^{2}}=-Z(\omega) \delta\left(x-x^{\prime}\right) \frac{\partial}{\partial t}\right] \psi(x, t \mid \omega)
$$

We assume the solution has a time dependence given by an exponential of angular velocity $\omega\left(\psi=\phi(x \mid \omega) \mathrm{e}^{-\mathrm{i} \omega t}\right)$ to obtain

$$
\left[\frac{\partial^{2}}{\partial x^{2}}+\kappa^{2}=\frac{-\mathrm{i} \omega}{E} Z(\omega) \delta\left(x-x^{\prime}\right)\right] \phi(x \mid \omega)
$$

where $\kappa=\omega / c_{l}$. Equation 5 can be solved analytically for a single scatterer or can be solved numerically for a particular system composed of a number of scatterers. From this numerical solution, one can determine the system resistivity, which can be used to calculate the localization length.

\section{CROSS SECTION}

The scattering cross section $\sigma$ for a single impurity can be calculated from the continuum system of Eqn. 5 The Green's function for the 1-D Helmholtz equation ${ }^{14}$ can be used to solve for the admittance $\phi(x)$ :

$$
\phi(x)=\mathrm{e}^{\mathrm{i} \kappa x}-\left(\frac{Z(\omega)}{Z(\omega)+\sqrt{4 \mu E}}\right) \mathrm{e}^{\mathrm{i} \kappa\left|x-x_{i}\right|} \mathrm{e}^{\mathrm{i} \kappa x_{i}}
$$

The scattering cross section in 1-D is equivalent to the reflection probability:

$$
\sigma=\frac{|Z(\omega)|^{2}}{|Z(\omega)|^{2}+4 \mu E}
$$

To apply this equation to the discrete chain, it must be converted from a continuum description to the corresponding discrete description.

For an FPU system having masses spaced a distance $a$ apart, the continuum coefficients can be expressed as a function of the discrete properties in the limit $a \rightarrow 0^{15}$ :

$$
\mu=\frac{m_{\circ}}{a} \quad E=K a
$$

The scattering cross section can now be expressed as a function of the discrete system components:

$$
\sigma=\frac{|Z(\omega)|^{2}}{|Z(\omega)|^{2}+4 K m_{\circ}}
$$

The impedance $Z(\omega)$ of a mass impurity along a onedimensional chain is proportional to the mass $m_{+}$that is added to the background mass $m_{0} 16$ :

$$
Z(\omega)=-\mathrm{i} \omega m_{+} / c_{s}
$$




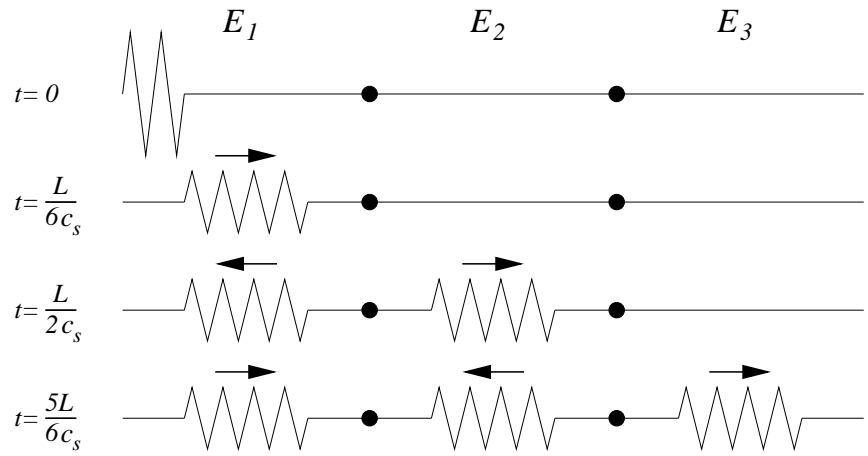

FIG. 1: Schematic of cross section numerical experiment; each line represents the state of the system at the time of measurement. The time $t$, shown along left side, is expressed as a function of the system length $L$ and pulse propagation speed $c_{s}$. The large dots denote the location of two impurities.

This equation has been modified from the continuum relation to account for the properties of a discrete onedimensional chain. A displacement wavelength has a corresponding wave number $k=2 \pi / \lambda$. For the discrete chain, the angular velocity $\omega$ and relative pulse propagation speed $c_{s}$ can be expressed as a function of the displacement wave number $k^{17}$ :

$$
\omega=2 \sin (k / 2) \quad c_{s}=\cos (k / 2)
$$

Finally, substituting Eqn.10]into Eqn.9gives the scattering cross section of a mass impurity in a one-dimensional chain:

$$
\sigma=\frac{\left(m_{+} \omega / c_{s}\right)^{2}}{\left(m_{+} \omega / c_{s}\right)^{2}+4 K m_{\circ}}
$$

A numerical experiment was performed to test the applicability of Eqn. 12 to FPU systems, and a schematic of the experiment is shown in Fig. 11 The system had fixed ends and length $L$. One impurity was located at $L / 3$, and another impurity was located at $2 L / 3$. An initial displacement was made with wavelength $\lambda$ and total length $16 \lambda$, and the hyperbolic tangent function was used to taper the oscillation amplitude from $A$ to zero. Initially, the pulse was located at one end of the system. It had length $8 \lambda$, amplitude $2 A$, and zero initial velocity. The time-dependent behavior was determined by numerical integration using the SIA4 algorithm.

The measured scattering cross section was determined from the energy located in the three regions separated by the impurities. The pulse energy located in each three regions, denoted by $E_{1}, E_{2}$, and $E_{3}$, was calculated at four separate times: $t=0, L / 6 c_{s}, L / 2 c_{s}$, and $5 L / 6 c_{s}$. At these times, the pulses are located near the center of a region. Although redundant with respect to the initial total energy, determining the energy at $t=L / 6 c_{s}$ provided a consistency check. In each case, the difference between $E_{1}(t=0)$ and $E_{1}\left(t=L / 6 c_{s}\right)$ was less than one part in $10^{4}$.

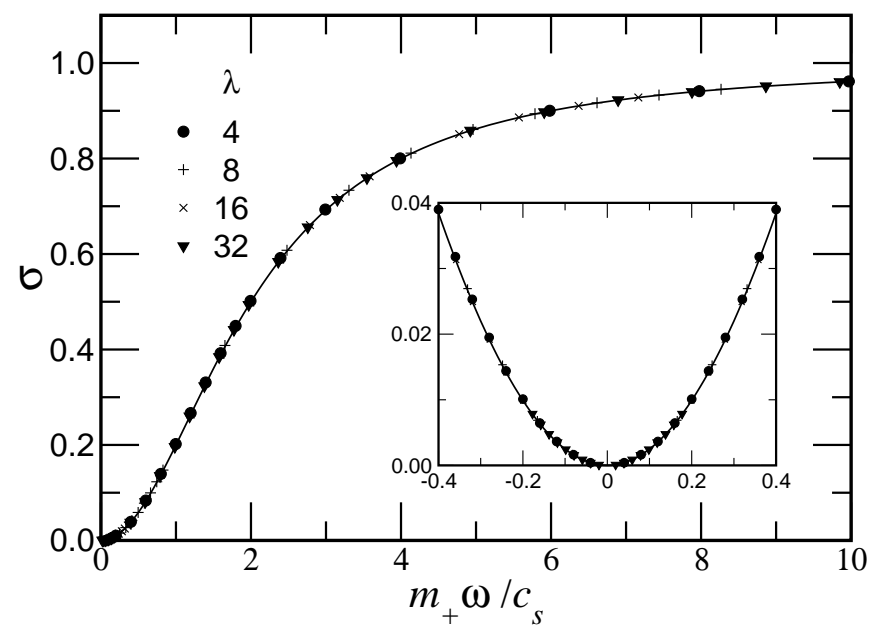

FIG. 2: Cross section $\sigma$ as a function of $m_{+} \omega / c_{s}$ for different wavelengths $\lambda$. The solid curve is the analytical result in Eqn. 12 The inset shows additional data near zero.

The scattering cross section in FPU systems is the reflection coefficient. In this experiment, the reflection coefficient $R$ is calculated from the ratio of energies in the first two intervals after one scattering event:

$$
R=\frac{E_{1}\left(L / 2 c_{s}\right)}{E_{1}(0)}
$$

Although the transmission coefficient $T$ could have been determined from $E_{2}\left(L / 2 c_{s}\right)$, a second scatterer was used as a more rigorous test of the experiment design and numerical integrator. The transmission coefficient $T$ for a single scatterer was calculated using the energy after two scattering events:

$$
T=\sqrt{\frac{E_{3}\left(5 L / 6 c_{s}\right)}{E_{1}(0)}}
$$

In all cases, the magnitude of $1-R-T$ was less than $10^{-3}$.

A comparison of the estimated cross section $\sigma$ in Eqn. 12 to the measured reflection coefficient $R$ in Eqn. 13 is shown in Fig. 2 for different displacement wavelengths $\lambda$. The results demonstrate that Eqn. 12 is an accurate estimate for the scattering cross section $\sigma$ for displacement wavelengths as short as 4. Moreover, the symmetry about zero for scattering cross section for negative values of $m_{+}$is shown in the inset of Fig. 2]

\section{LOCALIZATION LENGTH}

The resistivity $\rho_{N}$ of a system composed of $N$ scatterers is the ratio of the system reflection coefficient $R_{N}$ to the system transmission coefficient $T_{N}{ }^{2,3}$ :

$$
\rho_{N}=\frac{R_{N}}{T_{N}}
$$


The localization length of the system is defined in an averaged sense. To achieve proper scaling, however, one defines its inverse, the Lyapunov exponent $\gamma$ :

$$
\gamma=\frac{\ln \left(1+\rho_{N}\right)}{L}
$$

More specifically, there is a distribution of values from an ensemble of systems. If the systems composing the ensemble are sufficiently large, the distribution of $\gamma$ values will be normal. The localization length $\xi$ for a system having length $L$ and $N$ scatterers is defined from the ensemble averaged Lyapunov exponent:

$$
\xi^{-1}=\langle\gamma\rangle=\frac{\left\langle\ln \left(1+\rho_{N}\right)\right\rangle}{L}
$$

Unless otherwise noted, the symbol $\gamma$ shall imply the ensemble averaged quantity.

\section{A. Strong Scatterers}

To perform numerical experiments on anharmonic systems of manageable length, the scatters will need to be relatively strong. Therefore, weak scattering results will not be applicable. Moreover, a means is needed to predict the averaged localization behavior of a system using only single scatterer information. Because the impurities are identical, the scaling law ${ }^{3}$ can be exploited to express system behavior as a function of the resistivity of a single scatterer $\rho$ :

$$
\left\langle\ln \left(1+\rho_{N}\right)\right\rangle=N \ln (1+\rho)
$$

Substituting from Eqn. 17 above yields an unbiased estimate for the ensemble averaged localization length (dilute limite) as a function of impurity concentration $c$ :

$$
\xi_{\circ}^{-1}=c\langle\ln (1+\rho)\rangle
$$

In the limit of weak scattering $(c, \rho \rightarrow 0)$, one recovers the expected result $\xi_{\circ}^{-1}=c \sigma=\Lambda^{-1}$, where $\Lambda$ is the classical mean free path.

To demonstrate both the effect of strong scatterers $(\sigma \rightarrow 1)$ and the accuracy of Eqn. 18, the ensemble average $\left\langle\ln \left(1+\rho_{N}\right)\right\rangle$ was calculated from 10000 systems, each having length $L$ and $N$ impurities. The displacement wavelength was 32 , and the scattering cross section of each impurity was $\sigma$. The results from the calculation are shown in Fig. 3 as a function of the impurity cross section $\sigma$. In the figure, the error bars represent the mean standard deviation, and many of the symbols lie upon one another. As can be seen, all the systems have the $\ln (1+\rho)$ dependence that deviates from the mean free path approximation $\left(\xi_{\circ}^{-1}=c \sigma\right)$ for cross sections greater than approximately 0.2 for all combinations of system size and number of scatterers.

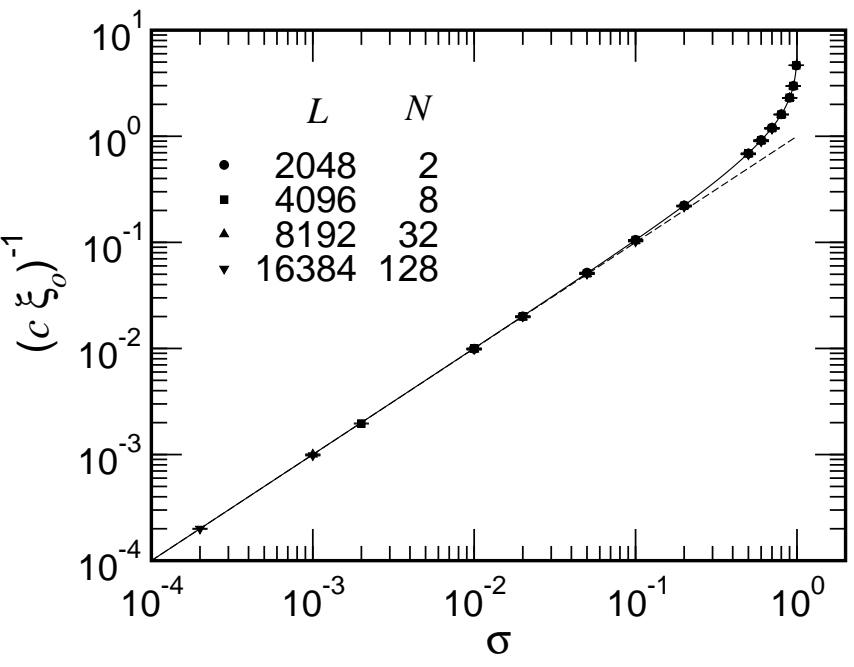

FIG. 3: Localization length $\xi_{\circ}$, scaled by impurity concentration $c$, as a function of impurity scattering cross section $\sigma$ for systems having length $L$ and $N$ impurities. The solid line is $\ln (1+\rho)$ and the dashed line represents the mean free path estimate. The error bars represent the mean standard deviation. (Many of the symbols lie upon one another and the error bars are typically smaller than the symbols.)

\section{B. Statistics}

The results shown in Fig. 3 demonstrate that, in the mean, systems having a finite density of scatterers have the expected behavior. Recent results suggest that systems having relatively few scatterers do not exhibit Gaussian behavior and, therefore, not obey scaling laws. For systems having sufficient length and number of scatterers, the population of Lyapunov exponents is normally distributed ${ }^{3}$, with variance $s_{\gamma}^{2} \underline{3.18}$

$$
s_{\gamma}^{2}=\frac{2}{L^{2}}\left\langle\ln \left(1+\rho_{N}\right)\right\rangle
$$

Returning to the data of Fig. 3. the population of Lyapunov exponents $\gamma$ was compared to the expectations of Eqn. 20 To assess the "normality" of the data, the intervals, both above and below the mean, having coverage factors 19 corresponding to one and two standard deviations were determined from the population. In addition, the population standard deviation $\Delta$ so that it could be compared to both its estimated value in Eqn. 20 and to the corresponding coverage interval.

The results of the Lyapunov exponent statistics calculation, from an ensemble with a population of 10000 , are shown in Fig. 4 for systems having length $L=16384$, $N=128$ impurities, and displacement wavelength $\lambda=$ 32. (Results for other systems were similar, and are omitted for brevity.) The data are shown as a function of the single impurity cross section $\sigma$. The filled symbols are the average value, and the solid line is the estimated average value. The error bars represent the intervals that have the same coverage factor as one and two standard devi- 


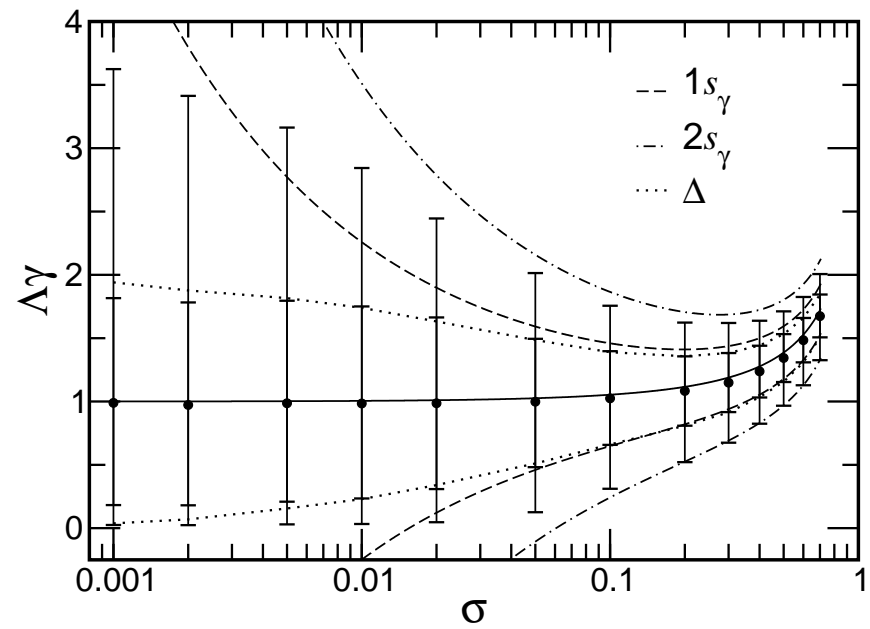

FIG. 4: Lyapunov exponent $\gamma$ statistics as a function of scattering cross section $\sigma$ for a system with $L=16384, N=128$, and $\lambda=32$. Error bars represent coverage factors corresponding to one and two standard deviations of a normal distribution. The predicted intervals $\pm 1 s_{\gamma}$ and $\pm 2 s_{\gamma}$ (Eqn. 20) are shown, along with observed population standard deviation $\Delta$.

ations in a normal distribution. The two pairs of dashed lines are the estimated standard deviations from Eqn. 20] The dotted line is the population standard deviation $\Delta$.

For small scattering cross sections, the distribution of $\gamma$ is asymmetric, with zero as a lower bound for the coverage intervals. Interestingly, only the outer intervals are asymmetric about the mean. The inner intervals are nearly symmetric about the mean, and they have a value nearly equal to the population standard deviation.

As the scattering cross section increases, the results begin to exhibit Gaussian behavior. Above a 0.2 scattering cross section, the measured intervals, the predicted intervals $s_{\gamma}$, and the population standard deviation $\Delta$ all agree. Therefore, agreement between the population standard deviation $\Delta$ and the estimated standard deviation $s_{\gamma}$ is as much as measure of "normality" as is a careful analysis of the population coverage intervals.

Exploiting this relationship, the population standard deviation $\Delta$ for all the data from the Lyapunov exponent statistics experiment are shown in Fig. [5]as a function of the system length. For normally distributed populations, the estimated coefficient of variation can be determined from Eqn. 177 and Eqn. 20 and has the following convenient form:

$$
\frac{s_{\gamma}}{\langle\gamma\rangle}=\sqrt{\frac{2}{L / \xi_{\circ}}}
$$

This equation is shown as a solid line in Fig. 5. One can conclude from the figure that in order for the observed Lyapunov exponents to be normally distributed, the system length must be at least 10 times the localization length, and the number of scatterers must be greater than approximately 32 .

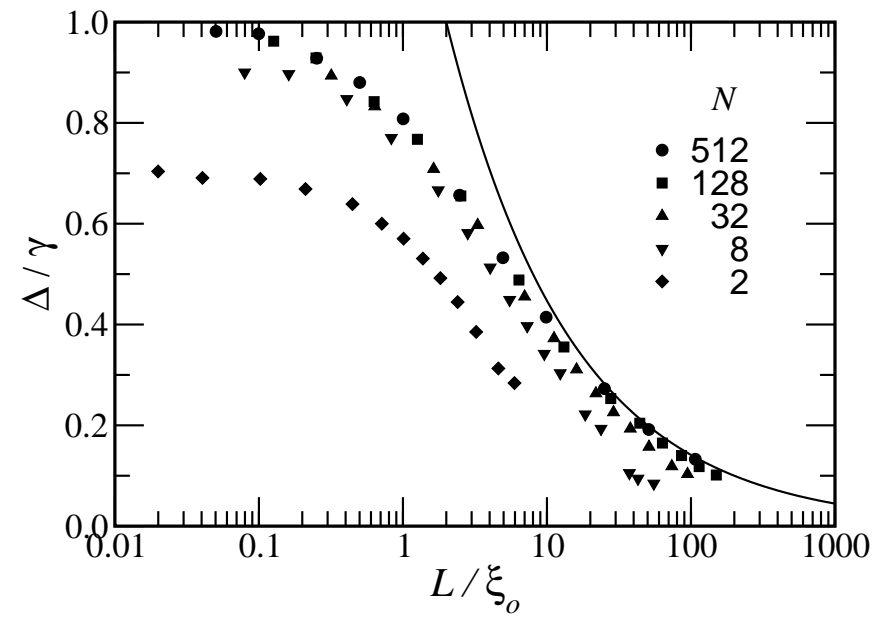

FIG. 5: Lyapunov exponent coefficient of variation $\Delta / \gamma$ as a function of the ratio of system length $L$ to the localization length $\xi_{\circ}$ for systems having different number of scatterers $N$. The solid curve is Eqn. 21]

\section{CONCENTRATED IMPURITIES}

For dilute impurity concentrations, the localization length decreases with increasing impurity concentration. As the impurity concentration $c$ approaches unity, however, the FPU system will become a pure system composed entirely of masses $m_{\circ}+m_{+}$. At $c=1$, the system is once again devoid of impurity and the localization length goes to infinity. At intermediate impurity concentrations, the localization length passes through a minimum. Therefore, the behavior of a system at arbitrary impurity concentration cannot be fully characterized by the relation in Eqn. [19]

For dilute systems, the localization length $\xi_{c \rightarrow 0}$ is as before:

$$
\xi_{c \rightarrow 0}^{-1}=\xi_{\circ}^{-1}=c\langle\ln (1+\rho)\rangle
$$

At high concentrations, the localization length $\xi_{c \rightarrow 1}$ has a concentration dependence that is proportional to $(1-c)$ :

$$
\xi_{c \rightarrow 1}^{-1}=(1-c)\left\langle\ln \left(1+\rho^{\prime}\right)\right\rangle
$$

The adjusted resistivity $\rho^{\prime}$ is for a system having background mass $m_{\circ}+m_{+}$and impurities with mass $m_{\circ}$. By the nature of the solution using the MK method, and given that the systems are harmonic, the frequency $\omega$ is the same for both systems, but the wavelength and the corresponding pulse speed are different:

$$
\begin{aligned}
k^{\prime} & =k \sqrt{m_{\circ}+m_{+}} \\
c_{s}^{\prime} & =\cos \left(k^{\prime} / 2\right) \\
\rho^{\prime} & =\frac{\left(-m_{+} \omega / c_{s}^{\prime}\right)^{2}}{4 K\left(m_{\circ}+m_{+}\right)}
\end{aligned}
$$

The behavior of the system for all values of impurity concentration is conjectured from the electrical analogy: 


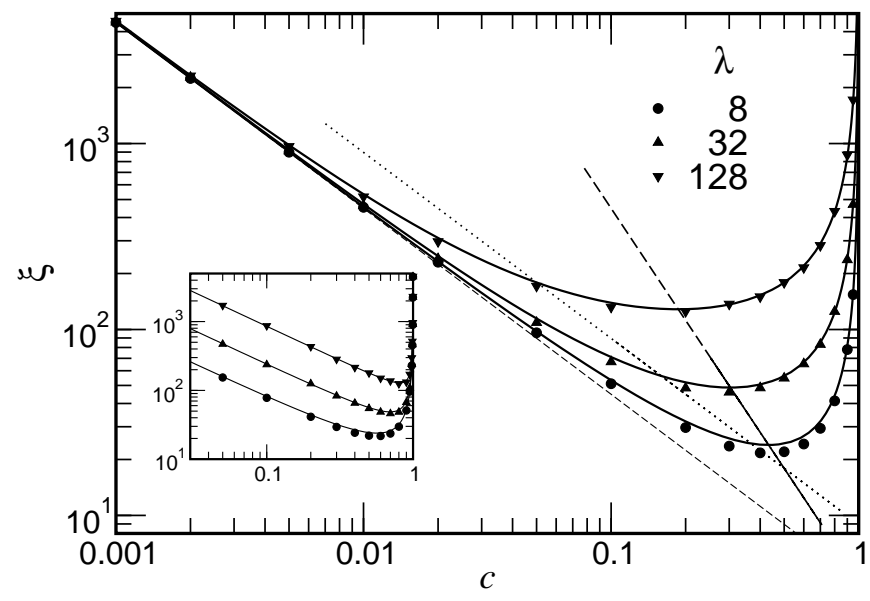

FIG. 6: Localization length $\xi$ as a function of impurity concentration $c$ in a discrete system having scatterers with cross section 0.2. Solid lines is Eqn. 27 dashed line is locus of minima, and dotted line denotes equal contribution from $\xi_{c \rightarrow 0}$ and $\xi_{c \rightarrow 1}$. Inset shows same data as a function of (1-c).

as Lyapunov exponent is to resistivity, localization length is to conductivity. If one assumes that at some intermediate concentration the behavior is simultaneously expressing itself as two systems with localization lengths $\xi_{c \rightarrow 0}$ and $\xi_{c \rightarrow 1}$, these two systems should contribute independently to the overall behavior. By analogy to conductors, the total localization length $\xi$ would be additive:

$$
\xi=\xi_{c \rightarrow 0}+\xi_{c \rightarrow 1}
$$

This equation represents a more complete estimate for the localization length that is valid for both strong and concentrated scatterers.

\section{A. Positive $m_{+}$}

The accuracy of this approximation is shown in Fig. 6 for systems having impurity scattering cross section $\sigma=$ 0.20 and displacement wavelengths ranging from 8 to 128 . The localization length $\xi$ for these systems was calculated using the MK solution. As can be seen, the approximation in Eqn. 27 is reasonably accurate. The inset shows the same data, plotted as a function of $(1-c)$, highlighting the separate behavior near $c \rightarrow 1$ for the different wavelengths.

Also shown in Fig. [6 are a dashed line and a dotted line. The dashed line is the locus of localization length minima as a function of displacement wavelength. Not only does the minimum localization length increase with increasing wavelength, the concentration at which this happens decreases with increasing wavelength. The dotted line in Fig. [6 is the locus of points where $\xi_{c \rightarrow 0}=\xi_{c \rightarrow 1}$. This locus of points is meant to delineate the cross-over point as the system passes from one dominate phase to the other. The cross-over point has a stronger concentration dependence than the locus of minima.

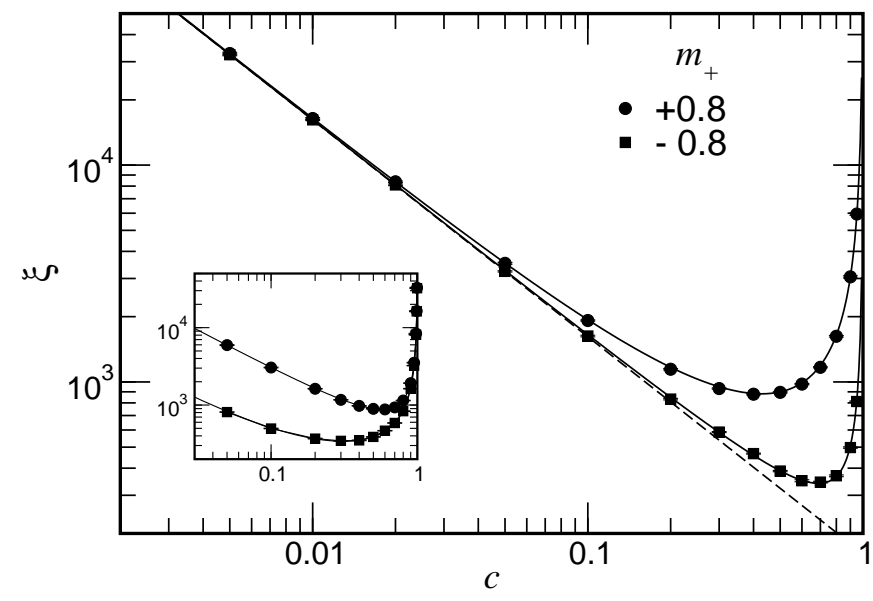

FIG. 7: Localization length $\xi$ as a function of impurity concentration $c$ for $m_{+}= \pm 0.8$. Inset shows same data as a function of $(1-c)$.

\section{B. Negative $m_{+}$}

At a given oscillation wavelength and frequency, the impurity scattering cross section depends upon the magnitude of the change in mass $m_{+}$. For small impurity concentrations, the localization length for a system with impurity mass $m_{\circ}+m_{+}$will be equal to one with impurity mass $m_{\circ}-m_{+}$. At higher concentrations, however, the behavior of systems will differ.

As an example, the localization concentration dependence was calculated for two systems with displacement wavelength $\lambda=32$. The added impurity masses were +0.8 and -0.8 , and the localization length was determined by the MK method. The results of the calculation are shown in Fig. 7 as a function of the impurity concentration $c$. The estimate from Eqn. 27 is shown as a solid line. Within the inset are the data plotted as a function of $(1-c)$. As expected, the behavior of the two systems diverge at higher concentrations.

\section{Azbel and Soven Comparison}

To better judge performance, Eqn. 27 is compared to the more rigorous result of Azbel and Soven ${ }^{6}$ (AS). The AS model contains quantum particles interacting with delta function potentials that have strength $V$ and are located at random integer values of $x$. For the AS systems, the value of $V$ is equal to -1 , and the scattering cross section $\sigma_{\mathrm{AS}}$ of each scatterer is a function of the particle wave number $k$ :

$$
\sigma_{\mathrm{AS}}=\frac{1}{1+16 \sin ^{2}(k / 2)}
$$

This is sufficient to duplicate the AS numerical calculation.

Figure 1 of Azbel and Soven ${ }^{6}$ shows results from calculations made for three values of wavenumber $k: 0.02$, 


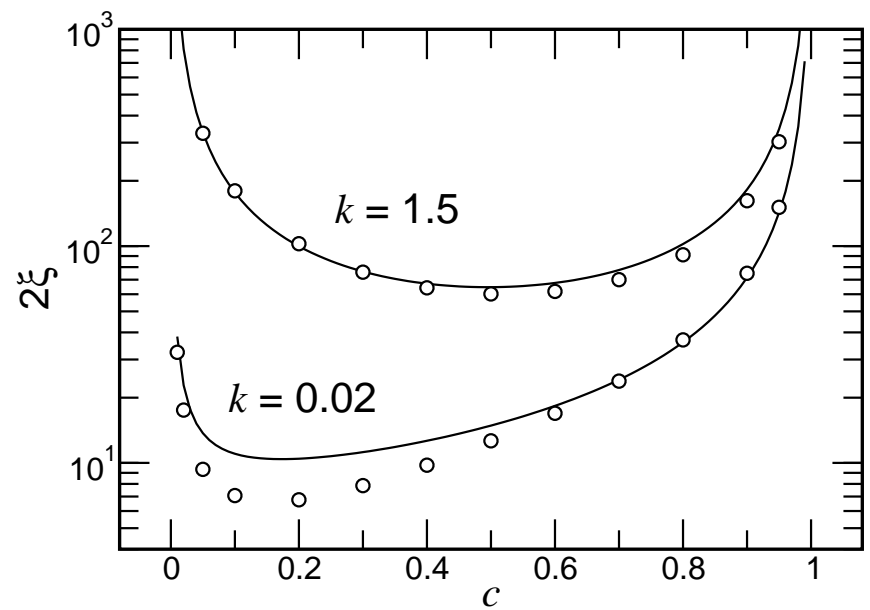

FIG. 8: Localization length $\xi$ as a function of impurity concentration $c$ for two systems with different wavenumber $k$. Open symbols are calculations using the MK method, and solid lines are Eqn. 27 The figure can be compared directly to Figure 1 of Azbel and Soven 6

1.5, 3.13. The MK method is used here to duplicate the numerical results for the two smaller values of $k$, and the results are shown as open symbols in Fig. [8] The solid lines in Fig. 8 are the corresponding estimate of Eqn. [27] Two things should be noted explicitly: The AS definition of localization length corresponds to twice the localization length defined here. The definition of localization length in Azbel and Soven ${ }^{6}$ uses the Landauer ${ }^{2}$ scaling parameter, while subsequent work ${ }^{20.21}$ use the scaling of Anderson et al $\stackrel{3}{.}$. The results shown in Fig. 8 use the latter scaling definitions.

\section{D. $c \lambda$ Effect}

As can be seen in Figs. [6] and [7] the behavior of the total localization length diverges from the dilute limit $\xi_{\mathrm{o}}$. In Fig. [6] the point at which $\xi$ begins to differ from $\xi_{\circ}$ is a function of the displacement wavelength. To more clearly demonstrate this effect, the ratio $\xi / \xi_{\circ}$ is shown in Fig. 9 as a function of the product $c \lambda$. The data shown are those appearing in Fig. 6] with the addition of those for $\lambda=$ 512. Also shown in the figure is a dotted line denoting the long wavelength limiting behavior for $\lambda \rightarrow \infty$. For values of $c \lambda$ greater than 1, the dilute limit approximation does not hold, and the observed localization length is greater than the dilute limit estimation. Therefore, even though the effect is less dramatic for increasing $\lambda$, there is a minimum effect, regardless of the wavelength.

\section{ANHARMONIC CHAINS}

In an anharmonic system, phonon-phonon interactions will lead to the creation of displacements with varying

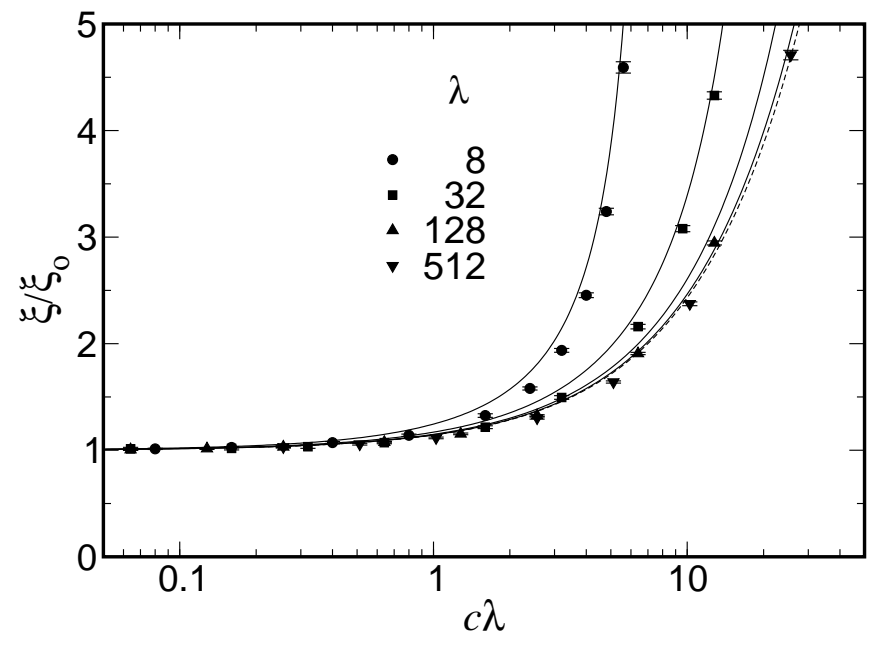

FIG. 9: The ratio of the measured localization $\xi$ to the calculated localization $\xi_{\circ}$ as a function of product of impurity concentration $c$ and wavelength $\lambda$. Filled symbols are calculated solutions for systems having impurity cross section 0.2 .

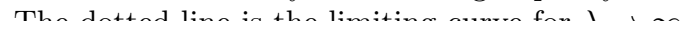



FIG. 10: The ratio of the measured localization $\xi$ to the dilute limit localization length $\xi_{\circ}$, normalized by the impurity concentration $c$, as a function of displacement wavelength $\lambda$.

wavelength. In time, very long wavelength displacements will be created. Because the impurity masses are fixed in time, the scattering cross section for the displacement waves will decrease as $\lambda^{-2}$. In addition, because the impurities are fixed in space, the product $c \lambda$ will increase over time. Based on the results from the last Section, one would expect that the ratio $\xi / \xi_{\text {o }}$ will diverge to infinity with increasing wavelength.

Using Eqn. 27 the ratio $\xi / \xi_{\circ}$ was calculated as a function of displacement wavelength for systems having constant impurity mass and concentration. The results are shown in Fig. 10 for different values for $m_{+}$. For all values of impurity concentration $c$, the ratio $\xi / \xi_{\circ}$ asymptotes to 
a constant at long wavelength:

$$
\lim _{\lambda \rightarrow \infty} \frac{\xi}{\xi_{\circ}}=1+\frac{c}{1-c}\left(m_{\circ}+m_{+}\right)
$$

Therefore, for anharmonic systems, the localization length is characterized, to within a constant, by the dilute limit expression for $\xi_{\text {o given in Eqn. [19] }}$

The same relationship applies to both positive and negative values for $m_{+}$. For negative values of $m_{+}$, in fact, in the limit $m_{+} \rightarrow-1$, the behavior of the system is accurately characterized by $\xi_{\circ}$ at long wavelengths.

\section{CONCLUSION}

The localization length for harmonic chains having binary disorder can be predicted accurately over a wide range of wavelengths, impurity cross section, and impurity concentration. The ingredient needed for this prediction is the cross section of a single scatterer, corrected for short wavelength displacements via the relative pulse propagation speed. The localization length over the entire impurity concentration range can then be estimated by approximating the system as a sum of two independent systems, each accounting for the behavior of the system at the two limits of impurity concentration.

The general result applies to systems in which the impurity mass is either larger or smaller that the original mass. Although the scattering cross section is symmetric about zero, with respect to the mass added to the background value, the localization length behavior differs for negative and positive changes in mass having the same scattering cross section. This difference in behavior with respect to localization length, along with previous results showing differences in the rate of phonon-phonon interactions, are discussed in the context of numerical experiments on anharmonic systems.

The general results also suggest that the localization length of long displacement wavelengths created by phonon-phonon interactions can be approximated, to within a constant, from dilute limit calculations results. For increasing concentration and constant impurity concentration, the localization length, with respect to the dilute limit prediction, will eventually diverge toward infinity. This deviation occurs for all wavelengths, and is a universal function of the impurity concentration and the displacement wavelength, for a constant cross section. By contrast, in a numerical experiment on an anharmonic system composed of fixed scatterers, the scattering cross section decreases with increasing wavelength. For these systems, the long wavelength behavior is, to within a constant, accurately predicted by dilute limit predictions. This conjecture will be confirmed in a future paper ${ }^{22}$.

\section{Acknowledgments}

This work was supported by the NSF under grant number DMR-01-32726.
1 P. W. Anderson, Phys. Rev. 109, 1492 (1958).

${ }^{2}$ R. Landauer, Philos. Mag. 21, 863 (1970).

3 P. W. Anderson, D. J. Thouless, E. Abrahams, and D. S. Fisher, Phys. Rev. B 22, 3519 (1980).

4 P. Sheng, B. White, Z.-Q. Zhang, and G. Papanicolaou, Phys. Rev. B 34, 4757 (1986).

${ }^{5}$ R. Bourbonnais and R. Maynard, Phys. Rev. Lett. 64, 1397 (1990).

6 M. Y. Azbel and P. Soven, Phys. Rev. Lett. 49, 751 (1982).

7 T. Held, I. Pfeiffer, and W. Kuhn, Phys. Rev. B 55, 231 (1997).

8 S. Rohmfeld, M. Hundhausen, L. Ley, N. Schulze, and G. Pensl, Phys. Rev. Lett. 86, 826 (2001).

${ }^{9}$ F. Widulle, J. Serrano, and M. Cardona, Phys. Rev. B 65, 075206 (2002).

10 R. de L. Kronig and W. G. Penney, Proc. Roy. Soc. Lond. A 130, 499 (1931).

11 A. MacKinnon and B. Kramer, Phys. Rev. Lett. 47, 1546 (1981).

12 E. Fermi, J. Pasta, and S. Ulam, Tech. Rep., Los Alamos Scientific Laboratory (1955).
13 J. Candy and W. Rozmus, J. Comput. Phys. 92, 230 (1991).

14 G. Arfken, Mathematical Methods for Physicists (Academic Press, New York, 1970).

15 H. Goldstein, Classical Mechanics (Addison-Wesley, Reading, MA, 1980), 2nd ed.

16 P. M. Morse and K. U. Ingard, Theoretical Acoustics (Princeton University Press, 1968).

17 C. Kittel, Introduction to Solid State Physics (Wiley, 1986), sixth ed.

18 L. I. Deych, A. A. Lisyansky, and B. L. Altshuler, Phys. Rev. B 64, 224202 (2001).

19 Guide to the Expression of Uncertainty in Measurement (International Organization for Standardization (ISO), Genève, Switzerland, 1993), ISBN 92-67-10188-9.

${ }^{20}$ M. Y. Azbel and P. Soven, Phys. Rev. B 27, 831 (1983).

${ }^{21}$ M. Y. Azbel, Phys. Rev. B 28, 4106 (1983).

${ }^{22}$ K. A. Snyder and T. R. Kirkpatrick (2004), (to be submitted). 